\title{
Structure, configuration and sizing of Ni nanoparticles generated by ultrafast laser ablation in different media
}

\author{
D. Muñetón Arboleda ${ }^{1}$, J. M. J. Santillán ${ }^{1,2}$, L. J. Mendoza Herrera ${ }^{1}$, M. B. Fernández van Raap ${ }^{3,4}$, \\ D. Muraca ${ }^{5}$, D. C. Schinca ${ }^{1,6}$ and L. B. Scaffardi ${ }^{1,6}$ \\ ${ }^{1}$ Centro de Investigaciones Ópticas (CIOp), (CONICET La Plata - CIC), Argentina \\ 2 Departamento de Química, Facultad de Ciencias Exactas y Naturales, UNCa, Catamarca, Argentina \\ ${ }^{3}$ Instituto de Física La Plata (IFLP), CONICET, La Plata, Argentina \\ ${ }^{4}$ Departamento de Física, Facultad de Ciencias Exactas, UNLP, La Plata, Argentina \\ ${ }^{5}$ Instituto de Física "Gleb Wataghin" (IFGW), Universidade Estadual de Campinas, Brazil \\ ${ }^{6}$ Departamento de Ciencias Básicas, Facultad de Ingeniería, UNLP, Argentina
}

\begin{abstract}
In recent years, nickel nanoparticles (NPs) have increased scientific interest because of their extensive prospects in catalysts, information storage, large-scale batteries and biomedicine.

Several works on Ni NPs generation by laser ablation have appeared in the literature in the last years, using different pulsed laser regimes and different media have been published recently.

In this work we analyze the characteristics of species, structure (bare core or core-shell), configuration and size distribution of NPs generated by fs pulse laser ablation over a Ni solid target in n-heptane and water. We explore the presence of NiO-Ni core-shell and hollow $\mathrm{Ni}$ (or air-Ni) NPs in the colloids obtained. These were experimentally characterized using AFM and TEM microscopy, as well as Optical Extinction Spectroscopy (OES). Extinction spectra were modeled using Mie theory through an appropriate modification of the complex experimental dielectric function, taking into account a size-dependent corrective term for each free and bound electron contribution. Experimental UVvisible-NIR spectra were reproduced considering a size distribution of bare core, hollow and core-shell structures NPs.

In both media, Ni NPs shape and size distribution agrees with that derived from TEM and AFM analysis.
\end{abstract}

Keywords: nickel colloidal suspensions, nickel dielectric function, optical extinction spectroscopy, femtosecond pulse laser ablation, hollow nanoparticles

\section{INTRODUCTION}

Due to the different characteristics of metals at the nanometric scale, it is important to study the influence of size and shape on physical, chemical and biological properties [1-5].

Nowadays, Ni NPs have important scientific interest because of their prospects in magnetic behavior [6-8], catalysis [9, 10], enhanced optical properties [11] and biomedicine [12]. Thus, it becomes important to find new ways to produce and characterize Ni NPs. A very interesting way to fabricate colloidal NPs is laser ablation synthesis. Different authors use several pulsed laser regimes and different media to generate Ni NPs. For example, Dudoitis et al. [13] synthetized Ni NPs in argon and air using a Nd:YAG laser working in two different pulsed regimes, $15 \mathrm{~ns}$ and $10 \mathrm{ps}$. Amoruso et al. [14] used 300 fs laser pulses focused on a Ni target in vacuum.

For the case of generation of Ni NPs via laser ablation in liquids, Jaleh et al. [15] reported the ablation of a pure Ni plate immersed in methanol with PVP as stabilizer agent using a high repetition Nd:YAG laser of $240 \mathrm{~ns}$ pulse width. Musaev et al. [16] synthetized Ni colloidal suspensions in water by nanosecond laser ablation employing a nitrogen laser of $50 \mathrm{~ns}$ pulsewidth.

In this work, we study the characteristics of structure (bare core or core-shell) and size distribution of Ni colloids produced by femtosecond laser ablation in n-heptane and water. We used optical extinction spectroscopy to analyze the characteristics of the generated colloidal suspensions. This technique has a very high statistics since it probes around $10^{10}$ particles yielding reliable qualitative and quantitative information of the sample. The theoretical analysis of

Plasmonics: Metallic Nanostructures and Their Optical Properties XIII, edited by Allan D. Boardman, Din Ping Tsai, Proc. of SPIE Vol. 9547, 95473J · ( 2015 SPIE · CCC code: 0277-786X/15/\$18 · doi: 10.1117/12.2190421 
the extinction spectra were made by Mie theory [17] employing the size-dependent dielectric function, which is calculated from the experimental complex bulk plus size correctives terms. We use this model to explore the extinction spectrum dependence with size, and in the case of core-shell NPs, we investigate this dependence with inner and outer radius.

We report the presence of NiO-Ni core-shell and hollow Ni NPs in the colloids synthesized by ultrafast laser ablation. AFM and TEM techniques are performed independently on the colloidal samples yielding size and structural results that agree with those obtained by OES.

\section{EXPERIMENTAL}

A Ti:Sapphire (Ti:Sa) laser (chirped pulsed amplification, CPA) system that emits pulses of $120 \mathrm{fs}$ is used to ablate a thick nickel solid disk of $1 \mathrm{~mm}$ immersed in milli-Q water or $\mathrm{n}$-heptane to obtain appropriate colloidal suspensions. The pulse energy used in this experiment was $100 \mu \mathrm{J}$ per pulse. The sample was moved using a XY motorized micrometric stage, to assure that the laser impinged always in different points of the sample during 9 min.

AFM images were registered in air, at room temperature, using standard semicontact-mode of an NT-MDT Solver Pro microscope. APPNANO ACTA and ACLA probes at resonant frequencies of $273 \mathrm{kHz}$ and $166 \mathrm{kHz}$ were used for samples in n-heptane and in water respectively. Rectangular shaped semicontact-mode cantilevers with tip curvature radius of $6 \mathrm{~nm}$ were used for topographical measurements. A drop of a diluted sample was placed on a freshly cleaved muscovite mica sheet V-1 grade (SPI Supplies) and dried for $12 \mathrm{~h}$ at room temperature. AFM analysis of mica sheets yielded an average roughness of $0.0612 \mathrm{~nm}$.

TEM images were performed with a TEM-MSC (JEOL 2100, acceleration voltage $200 \mathrm{KV}$ ) at Brazilian Nanotechnology National Laboratory (LNNano). The as-prepared Ni colloid was diluted in n-heptane or mili-Q water and sonicated during $15 \mathrm{~min}$.

OES was conducted by means of a Shimadzu spectrophotometer from $250 \mathrm{~nm}$ to $1000 \mathrm{~nm}$. It was performed on colloidal suspensions immediately after fabrication, preventing possible NPs coalescence.

\section{EXTINCTION SPECTRA CALCULATION}

Although $\mathrm{Ni}$ is a ferromagnetic metal, for single domain NPs, the complex susceptibility $\chi(\omega)$ is inversely proportional to the external field frequency. For the visible range $\left(10^{14}-10^{15} \mathrm{~Hz}\right)$, the value of $\chi(\omega)$ is very close to zero and therefore, the relative permeability is unity $\left(\mu_{r}=1\right)$, allowing the NP extinction to be correctly described by Mie theory [17] for nonmagnetic NPs.

For the general case of coated spherical particles with core radius $R$ and outer radius $R^{\prime}$, the extinction cross section can be expressed as:

$$
C_{\text {ext }}=\frac{2 \pi}{k^{2}} \operatorname{Re}\left\{\sum_{n=1}^{\infty}(2 n+1)\left(a_{n}+b_{n}\right)\right\}
$$

where $a_{n}$ and $b_{n}$ are the scattering coefficients which depend on the first, second and third order spherical Bessel functions. The argument of these functions depends on the core and shell size parameters and their dielectric functions. The extinction efficiency is defined as $Q_{\text {ext }}=C_{\text {ext }} / \pi R^{\prime 2}$.

The experimental bulk complex dielectric function $\left(\varepsilon_{b u l k}(\omega)\right)$ of metals can be written as an additive contribution of free and bound electrons. When the size of metal NPs is smaller than the electron mean free path, the expression may be rewritten adding size corrective terms for free and bound electrons:

$$
\varepsilon_{\text {size }}(\omega, R)=\varepsilon_{\text {bulk }}(\omega)+\delta \varepsilon_{\text {free }}(\omega, R)+\delta \varepsilon_{\text {bound }}(\omega, R)
$$

where

$$
\delta \varepsilon_{\text {free }}(\omega, R)=\omega_{p}^{2}\left(\frac{1}{\omega^{2}+i \gamma_{\text {free }} \omega}-\frac{1}{\omega^{2}+i \gamma_{\text {free }} \omega+i C \omega \frac{v_{F}}{R}}\right)
$$


and

$$
\delta \varepsilon_{\text {bound }}(\omega, R)=-e^{-\left(\frac{R}{R_{o}}\right)}\left(\varepsilon_{\text {bulk }}(\omega)-1+\frac{\omega_{p}^{2}}{\omega^{2}+i \gamma_{\text {free }} \omega}\right)
$$

Here, $\omega_{p}$ is the bulk plasma frequency and $\gamma_{\text {free }}$ is the damping constant of the electron oscillatory movement due to electron-electron, electron-ion and electron-phonon collisions. In equation (3) $v_{F}$ is the Fermi velocity, $C$ is a constant that depends on the material and on the electron scattering processes at the particle boundary.

Figure 1 shows the real and imaginary parts of $\mathrm{Ni}$ size-dependent dielectric function versus wavelength for different radii considering the bulk dielectric function data given by Rakić et al. [18]. Theoretical calculations were performed based on equations (2) to (4), using $C=0.8[19], v_{F}=2.8 \times 10^{14} \frac{\mathrm{nm}}{\mathrm{s}}$ [20], $R_{O}=0.35 \mathrm{~nm}$ [21], $\omega_{p}=(6.41 \pm 0.12) \times 10^{15} \frac{1}{\mathrm{~s}}$ and $\gamma_{\text {free }}=(3.16 \pm 0.35) \times 10^{13} \frac{1}{\mathrm{~s}}$. The last two parameters were determined using the method described by Mendoza et al [22].
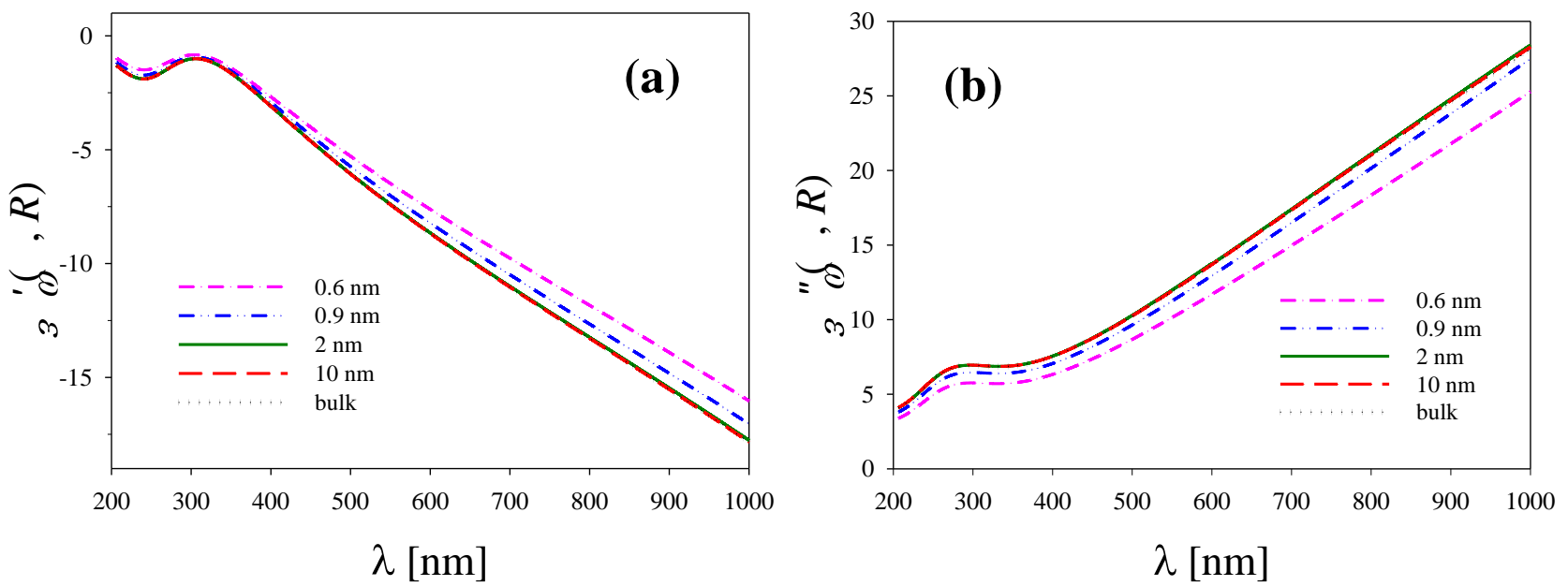

Figure 1. Calculated values of (a) real and (b) imaginary parts of Ni size-dependent dielectric function vs wavelength for different radii using equations (2) to (4).

The experimental bulk dielectric function is represented by the curve in dotted line. The curve for $2 \mathrm{~nm}$ radius (full line) is almost superimposed to that for bulk, indicating that for radii larger than $2 \mathrm{~nm}(R>2 \mathrm{~nm})$, size corrections are negligible. However, for sizes smaller than $2 \mathrm{~nm}(R<2 \mathrm{~nm})$, curves depart from the bulk dielectric function in the considered wavelength range between $200 \mathrm{~nm}$ and $1000 \mathrm{~nm}$.

Since the primary result of fs laser ablation in liquids is the production of a colloidal suspension, a spectrophotometric analysis may be readily performed on the vial. To fit this result, it is necessary to have knowledge of the extinction spectrum for different kinds of spherical NPs. Figure 2 (a) shows the extinction efficiency $Q_{\text {ext }}$ of bare Ni NPs in water for radii between $2 \mathrm{~nm}$ and $20 \mathrm{~nm}$, plotted as a function of wavelength using equation (1). For $7 \mathrm{~nm}$ radius a plasmon resonance can be observed as a small shoulder on the extinction curve. When the radius increases, this resonance is progressively redshifted up to $550 \mathrm{~nm}$. For bare Ni NPs in n-heptane a similar redshift in plasmon resonance is observed. 

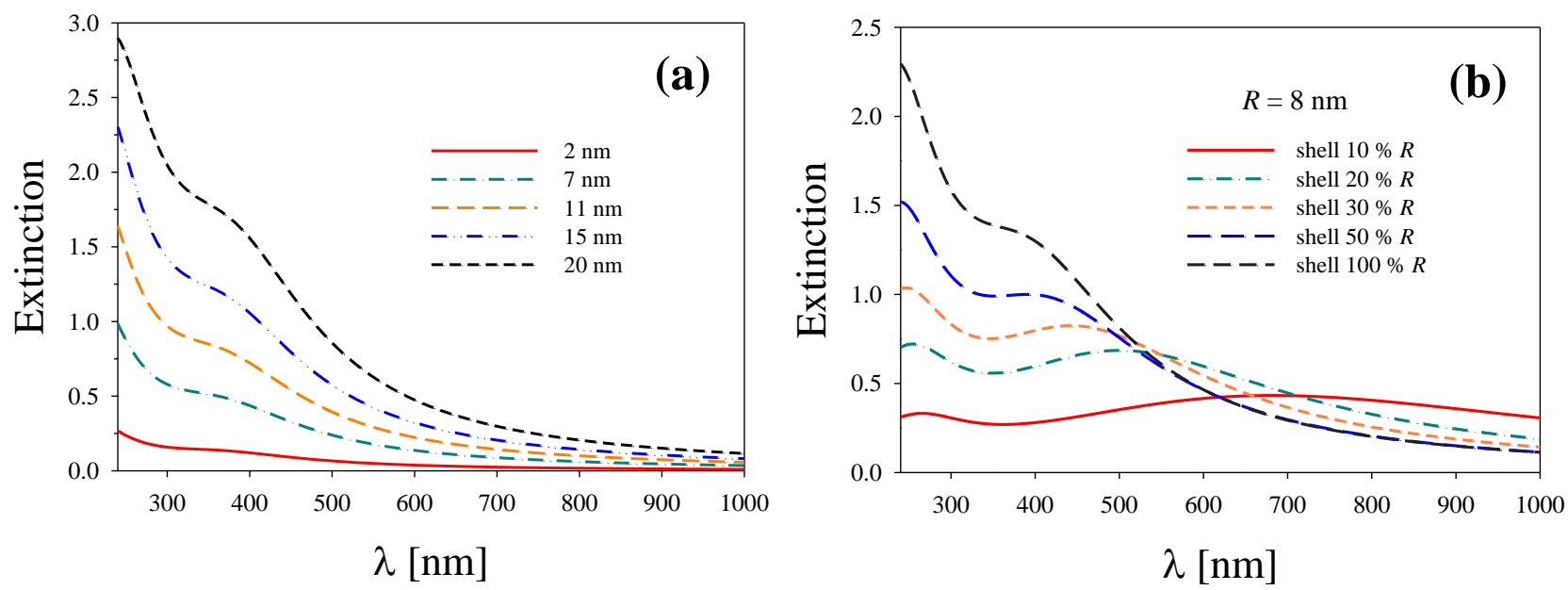

Figure 2. (a) Extinction spectra of Ni bare core NPs in water. (b) Extinction spectra for core-shell air-Ni NPs in water for core radius $R=8 \mathrm{~nm}$ and different shell thicknesses.

For core-shell type NPs, extinction efficiency depends on the inner radius $R$ and the outer radius $R^{\prime}$. This dependence in outer radius is shown in Figure 2 (b) for core-shell air-Ni NPs in water for a fixed core radius $R=8 \mathrm{~nm}$. The plasmon resonance that appears around $400 \mathrm{~nm}$ for $100 \%$ shell thickness redshifts as the Ni shell thickness decreases.

Oxidation-reduction processes may occur during laser ablation of certain metals in water. This is the case of $\mathrm{Ni}$ $[23,24]$. Under these circumstances, it is appropriate to consider the possibility of generating core-shell NPs of the type $\mathrm{Ni}-\mathrm{NiO}$ and NiO-Ni. Extinction spectra of core-shell Ni-NiO and NiO-Ni NPs in water for a core radius $R=8 \mathrm{~nm}$ for different percentages of shell thicknesses are shown in Figure 3 (a) and (b) respectively. Plasmon resonance is strongly redshifted as the shell thickness decreases.
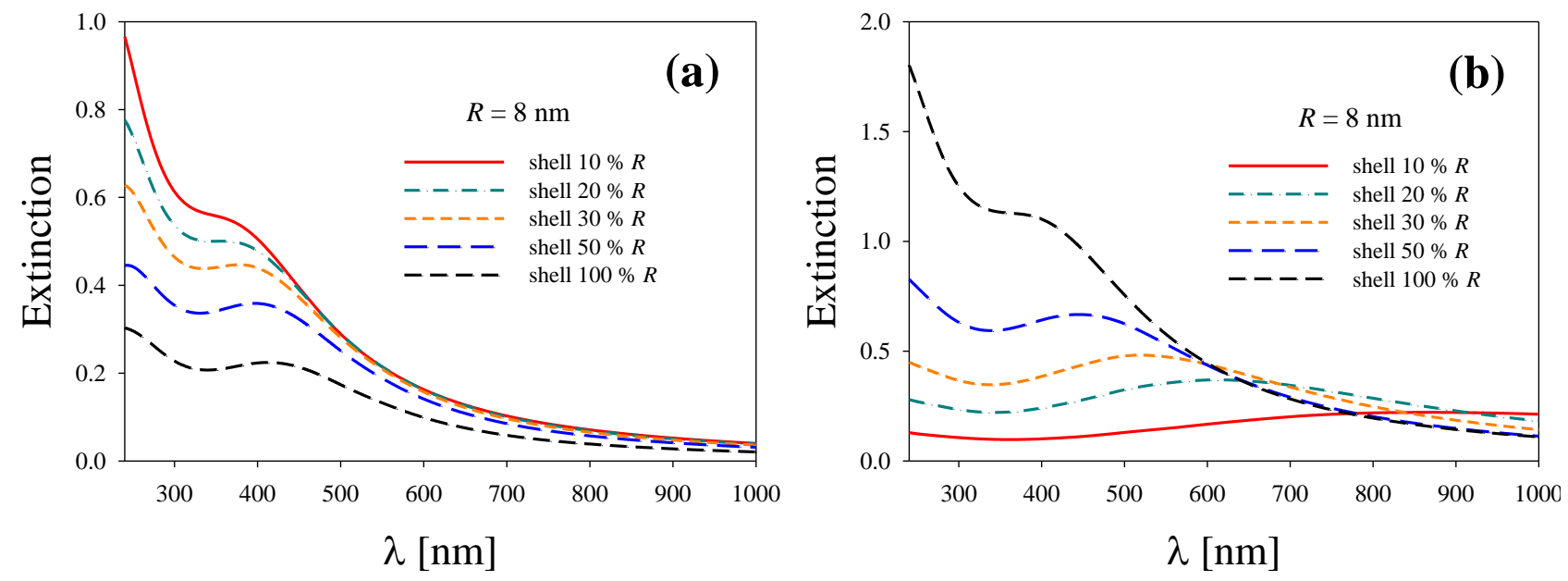

Figure 3. Optical extinction spectra calculated for (a) $\mathrm{Ni}-\mathrm{NiO}$ and (b) NiO-Ni core-shell NPs in water, for core radius $R=8 \mathrm{~nm}$ and different shell thicknesses.

Similar displacements with shell thickness are observed when $n$-heptane is the surrounding media. 


\section{EXPERIMENTAL RESULTS}

Figure 4 (a) and (b) show experimental extinction spectra in the UV-visible-near IR range for Ni colloidal suspensions fabricated by fs laser ablation in n-heptane and water, respectively. Full line represents the experimental spectrum while dotted line corresponds to the theoretical fit according to the size distribution shown in each inset, which are determined using equations (1)-(4). In panel (a), particle sizing is represented by log-normal size distributions of bare core Ni NPs with modal radii at $2.5 \mathrm{~nm}$ and $10 \mathrm{~nm}$ together with hollow Ni NPs at external modal radii of $6.6 \mathrm{~nm}, 12 \mathrm{~nm}$ and $15.6 \mathrm{~nm}$. For the case of water, panel (b), some degree of oxidation is surely to occur as we mentioned above. Structures and configurations like bare core $\mathrm{NiO}$ besides core-shell structures of Ni-NiO, NiO-Ni as well as hollow Ni were considered to be present in the colloidal suspension. The inset shows the total size distribution (full line) that yields the optimum fit of the experimental spectrum. The multimodal size distribution shape is similar to that determined for n-heptane but shifted to larger values of external radii. This fact may be due to the presence of oxide shells around the NPs.
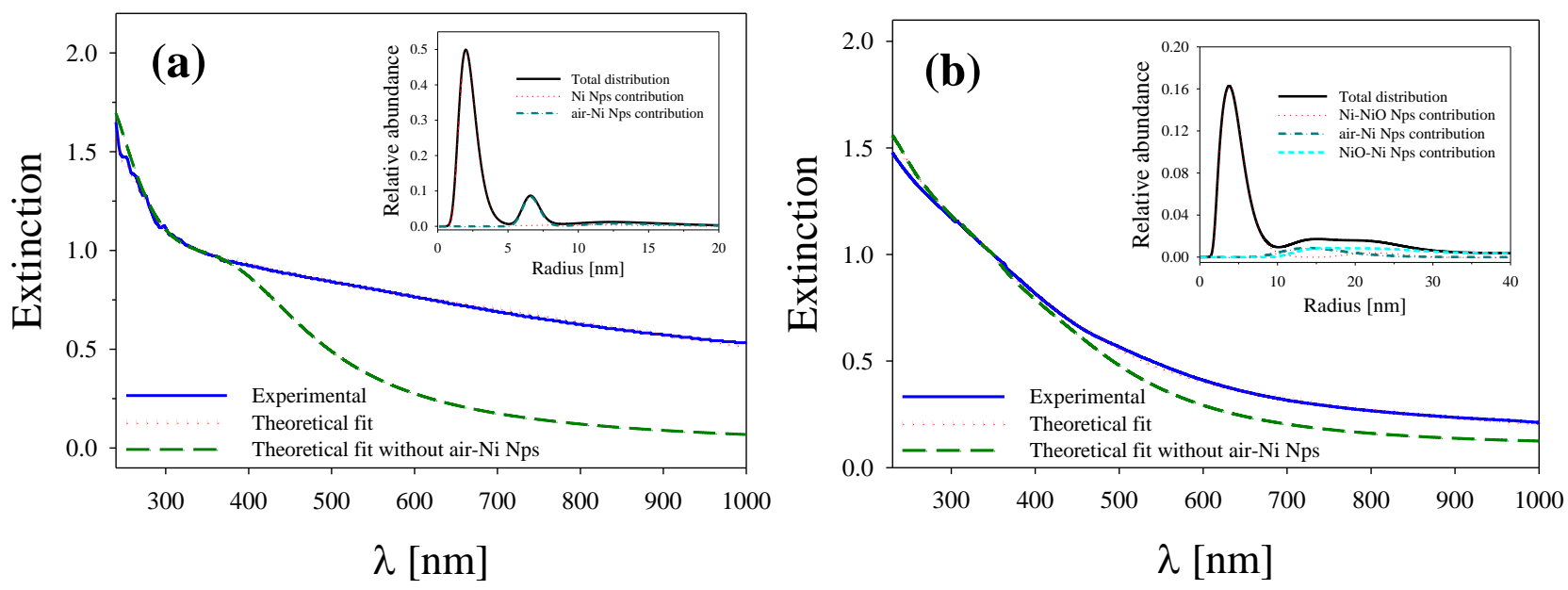

Figure 4. Comparison between experimental (full line) and theoretical (dotted line) extinction spectra of Ni colloidal suspensions in: (a) n-heptane and (b) water. The insets show size distributions of: (a) bare core and air-Ni relative abundance contributions that fit experimental spectrum and (b) Ni-NiO, air-Ni and NiO-Ni NPs that fits experimental spectrum. Long dashed line represents calculated spectrum with the same distribution without taking into account air-Ni NPs.

In both cases, hollow-type NPs must be considered for the accurate fit of the experimental extinction spectrum. Calculated extinction without considering this contribution misses to fit the experimental spectrum from $380 \mathrm{~nm}$ onward.

It is interesting to note that from the theoretical fit of the extinction spectra it is possible to determine the different species in the colloidal suspensions generated in $n$-heptane and water. For the first case, two species of NPs are present: $\mathrm{Ni}$ and hollow $\mathrm{Ni}$, while for water four species are present: $\mathrm{NiO}, \mathrm{Ni}-\mathrm{NiO}, \mathrm{NiO}-\mathrm{Ni}$ and hollow $\mathrm{Ni}$.

Shape and size of the Ni NPs synthesized were also characterized using AFM images. For n-heptane, panel (a) of Figure 5 shows a $45 \mu \mathrm{m} \times 35 \mu \mathrm{m}$ scanned area image where small single NPs are observed. Height profiles of selected scanned lines are shown in panel (b). NPs with typical external diameters of $23.7 \mathrm{~nm}, 19.7 \mathrm{~nm}, 13.7 \mathrm{~nm}, 5 \mathrm{~nm}$ and $32.1 \mathrm{~nm}$ respectively were observed. 

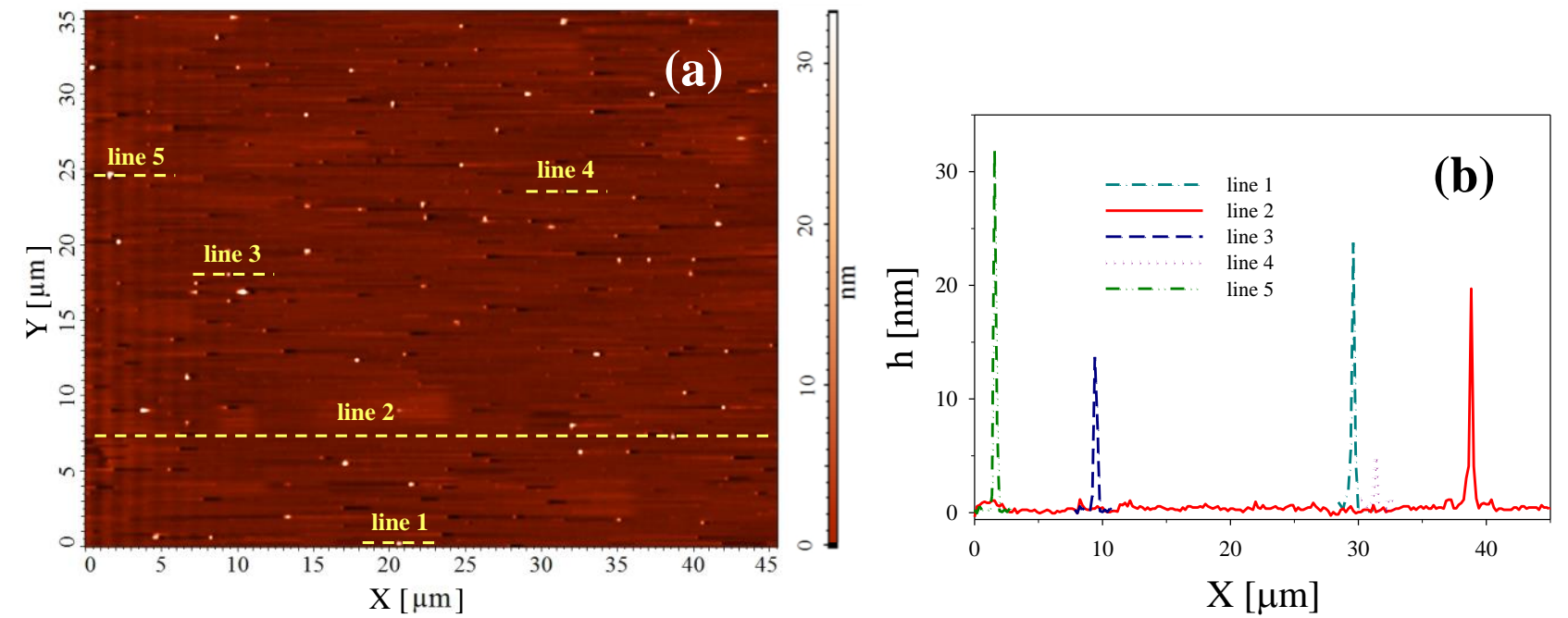

Figure 5. AFM size analysis of the obtained Ni colloidal suspension in n-heptane: (a) image of single Ni NPs; (b) height profiles of selected lines 1 to 5 from panel (a) which correspond to the spherical particles diameter.

These height profiles are in good agreement with the external radii distribution determined from the theoretical fit of the extinction spectrum of the sample shown in the inset of Figure 4 (a).

Figure 6 (a) shows a $30 \mu \mathrm{m} \times 30 \mu \mathrm{m}$ scanned area AFM image showing single NPs from the water colloidal suspension dried on fresh mica surface. Height profiles scanned in lines 1 to 6 show NPs with typical external diameters of $9.5 \mathrm{~nm}, 51.8 \mathrm{~nm}, 76 \mathrm{~nm}, 6.5 \mathrm{~nm}, 31.4 \mathrm{~nm}$ and $42.1 \mathrm{~nm}$ respectively, as indicated in Figure 6 (b).
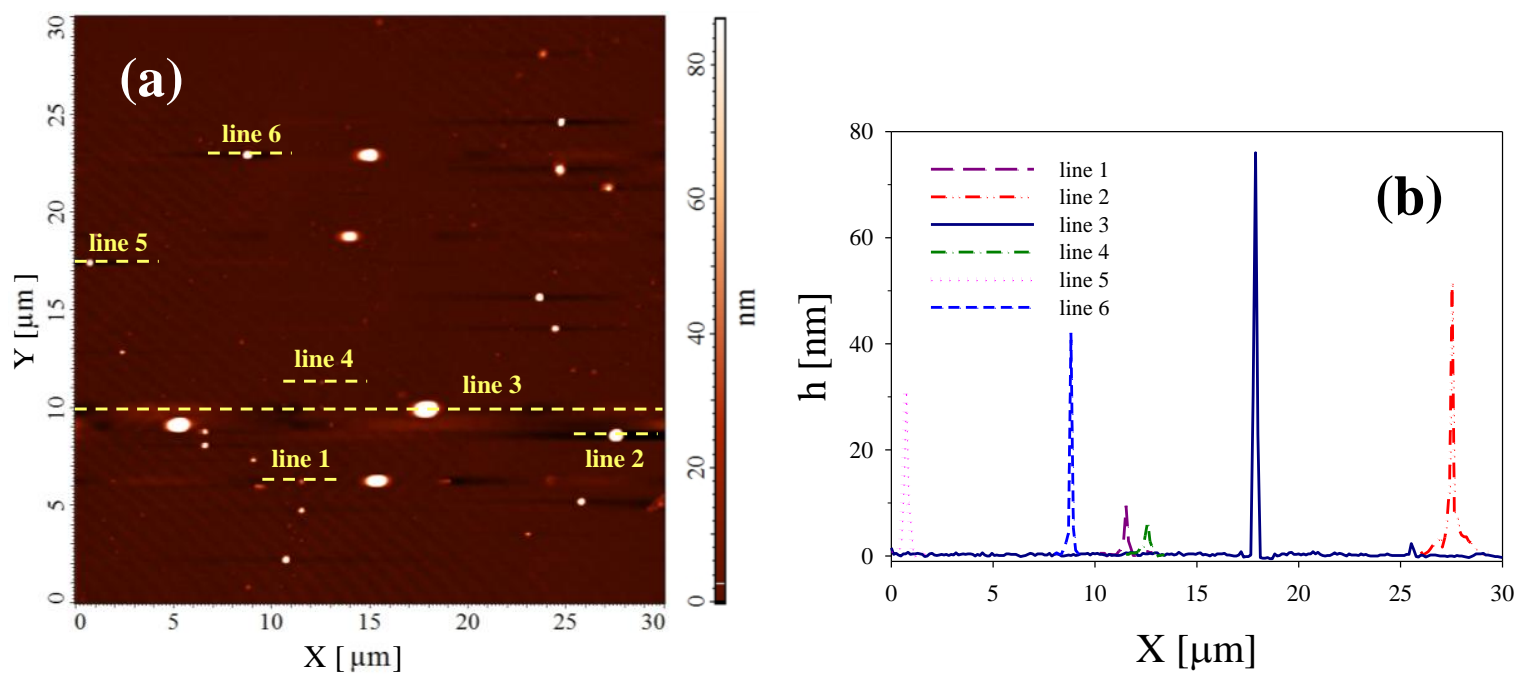

Figure 6. AFM size analysis of the obtained NPs after ablation of Ni target in water: (a) image of single NPs; (b) height profiles of selected lines 1 to 6 from panel (a).

As in the case of $n$-heptane, there is a good agreement between the external radii distribution obtained by OES and height profiles provided by AFM.

On the other hand, the morphology of the obtained Ni NPs, was studied using TEM analysis. Figure 7 shows TEM images of Ni colloidal suspensions in n-heptane (panel (a)) and water (panels (b) and (c)). The panoramic view 
contains isolated bare core and hollow Ni NPs is shown in panel (a). Dotted line outlines the limit of the core and shell structure.

When solid $\mathrm{Ni}$ is ablated in water, somewhat different results are obtained. Figure 7 (b) and (c) shows TEM images of Ni colloidal suspension obtained. Panel (b) shows an isolated hollow Ni NP in the bottom right corner and immediately above can be observed a Ni-NiO core-shell structure. Panel (c) contains a large size bare core NP.

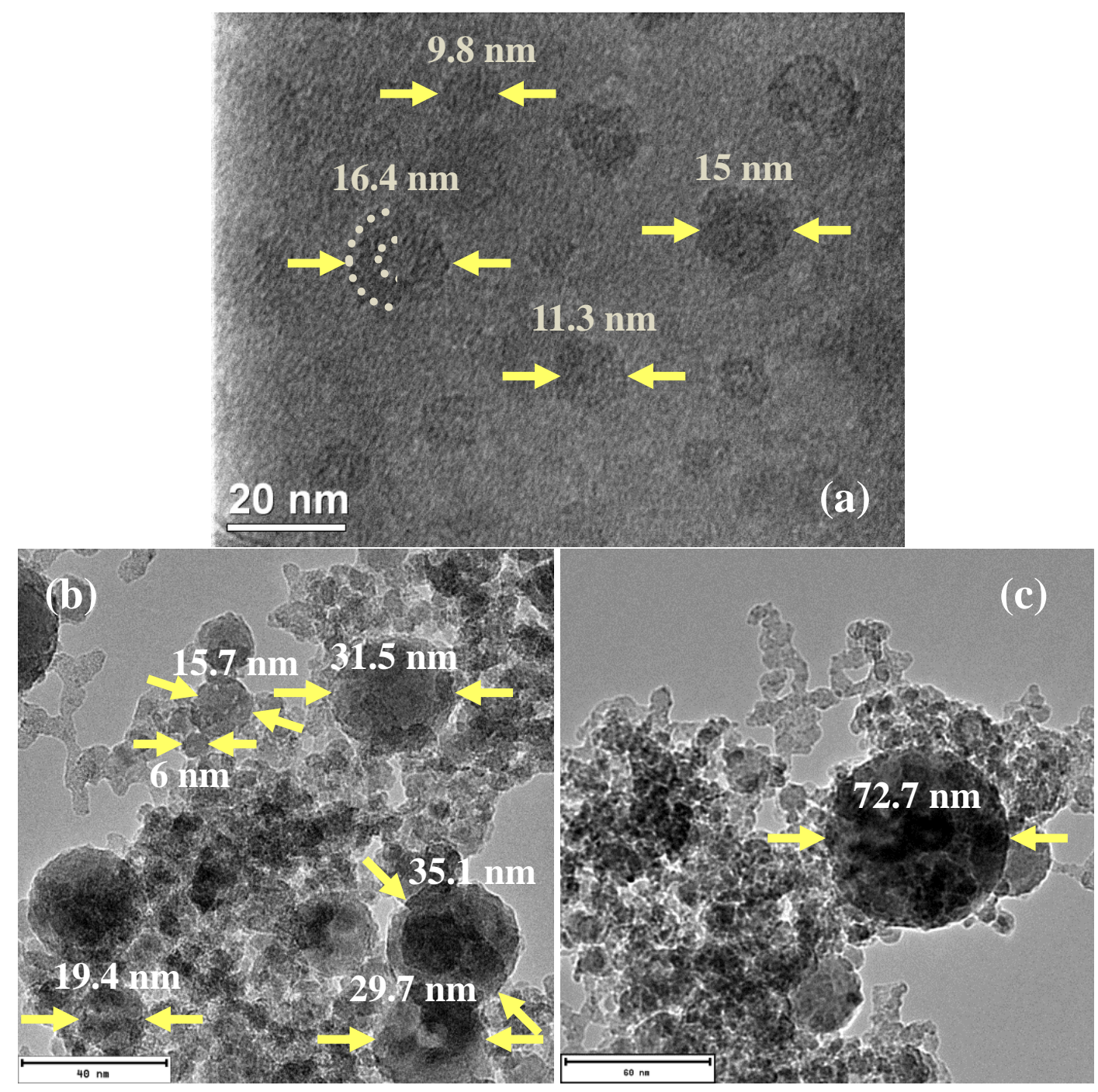

Figure 7. TEM images of NPs present in the Ni colloidal suspensions in: (a) n-heptane, (b) and (c) water. (a) Panoramic view which includes bare core and hollow Ni NPs. (b) Single hollow Ni (29.7 nm diameter) and single Ni-NiO (35.1 nm diameter) core-shell NPs and (s) spherical bare core larger diameter NP can be observed.

For both solvents, there is a good agreement between TEM, AFM and OES results. 


\section{CONCLUSIONS}

We generated Ni colloidal suspensions in n-heptane and water implemented by fs pulsed laser ablation on solid target. The NPs were analyzed using spectroscopic and microscopy (AFM and TEM) techniques to characterize their structure and size. The observation by microscopy shows that NPs were spherical in shape with structures: bare core and core-shell.

By the fit of the experimental extinction spectra for both solvents using Mie theory with appropriate size correction of the bulk complex dielectric function allowed the determination of the size distributions of bare core and core-shell NPs in the suspension. For n-heptane, the distribution consists of two different structures: bare core and hollow Ni NPs. For water the presence of oxygen in water favors the formation of oxide species, yielding the existence of core-shell structures $\mathrm{Ni}-\mathrm{NiO}$ and $\mathrm{NiO}-\mathrm{Ni}$, in addition to the hollow structure found above.

The results of the presence of spherical bare core, metal-metal oxide core-shell structures and hollow Ni NPs within the suspension is confirmed by the TEM analysis

TEM and AFM offer a very local analysis of the sample, while OES has a greater statistics than the first two. For this reason OES yields reliable results about size distribution and structures.

\section{ACKNOWLEDGMENTS}

This work was granted by PIP 0394 and PIP 0720 of CONICET, PME2006-00018 of ANPCyT, grant 11/I151 of Facultad de Ingeniería Universidad Nacional de La Plata and grant 11/X680 of Facultad de Ciencias Exactas Universidad Nacional de La Plata, Argentina. We thank C2NANO - Brazilian Nanotechnology National Laboratory (LNNano) at Centro Nacional de Pesquisa em Energia e Materiais (CNPEM) / MCT (\#14825 and 14827) and Research Proposal TEM-16976 for the use of TEM. AFM was carried out at LFAyM of Instituto de Física La Plata (IFLP CONICET).

D. C. Schinca is Member of Comisión de Investigaciones Científicas de la Provincia de Buenos Aires (CIC). L. B. Scaffardi, M. B. Fernández van Raap and J. M. J. Santillán are researchers of CONICET. D. Muraca is Member of Instituto de Física "Gleb Wataghin" (IFGW), Campinas, Brazil. D. Muñetón Arboleda and L. J. Mendoza Herrera are $\mathrm{PhD}$ fellows of CONICET, Argentina.

\section{REFERENCES}

[1] Chen, C-C, Herhold, A. B., Johnson, C. S. and Alivisatos, A. P. "Size Dependence of Structural Metastability in Semiconductor Nanocrystals," Science, 276, 398-401 (1997).

[2] Andrade, A. L., Valente, M. A., Ferreira, J. M. F. and Fabris, J. D. "Preparation of size-controlled nanoparticles of magnetite," J. Magn. Magn. Mater., 324, 1753-1757 (2012).

[3] Hou, Y. and Gao, S. "Monodisperse nickel nanoparticles prepared from a monosurfactant system and their magnetic properties," J. Mater. Chem., 13, 1510-1512 (2003).

[4] Sun, S., Murray, C. B., Weller, D., Folks, L. and Moser, A. "Monodisperse FePt Nanoparticles and Ferromagnetic FePt Nanocrystal Superlattices," Science, 287, 1989-1992 (2000).

[5] Beecroft, L. L. and Ober, C. K. "Nanocomposite Materials for Optical Applications," Chem. Mater., 9, 1302-1317 (1997).

[6] Couto, G. G., Klein, J. J., Schreiner, W. H., Mosca, D. H., de Oliveira, A. J. A. and Zarbin, A. J. G. "Nickel nanoparticles obtained by a modified polyol process: Synthesis, characterization, and magnetic properties," J. Colloid Interface Sci., 311, 461-468 (2007).

[7] Ramírez-Meneses, E., Betancourt, I., Morales, F., Montiel-Palma, V., Villanueva-Alvarado, C. C. and HernándezRojas, M. E. "Superparamagnetic nickel nanoparticles obtained by an organometallic approach," J. Nanopart. Res., 13, 365-374 (2011).

[8] Cheng, J., Zhang, X. and Ye, Y. "Synthesis of nickel nanoparticles and carbon encapsulated nickel nanoparticles supported on carbon nanotubes," J. Solid State Chem., 179, 91-95 (2006). 
[9] Park, J., Kang, E., Son, S. U., Park, H. M., Lee, M. K., Kim, J., Kim, K. W., Noh, H.-J., Park, J.-H., Bae, C. J. and Park, J.-G. "Hyeon, T. Monodisperse Nanoparticles of Ni and NiO: Synthesis, Characterization, Self-Assembled Superlattices, and Catalytic Applications in the Suzuki Coupling Reaction," Adv. Mater., 17, 429-434 (2005).

[10] Xu, R., Xie, T., Zhao, Y. and Li, Y. "Quasi-homogeneous catalytic hydrogenation over monodisperse nickel and cobalt nanoparticles," Nanotechnology, 18, 055602-055605 (2007).

[11] Murugadoss, G. and Rajesh Kumar, M. "Synthesis and optical properties of monodispersed Ni2+-doped ZnS nanoparticles," Appl. Nanosci., 4, 67-75 (2014).

[12] Rodríguez-Llamazares, S., Merchán, J., Olmedo, I., Marambio, H. P., Muñoz, J. P., Jara, P., Sturm, J. C., Chornik, B., Peña, O., Yutronic, N. and Kogan, M. J. "Ni/Ni Oxides Nanoparticles with Potential Biomedical Applications Obtained by Displacement of a Nickel-Organometallic Complex," J. Nanosci. Nanotechnol., 8, 3820-3827 (2008).

[13] Dudoitis, V., Ulevičius, V., Račiukaitis, G., Špirkauskaite, N. and Plauškaitė, K. "Generation of metal nanoparticles by laser ablation," Lithuanian J. Phys., 51, 248-259 (2011).

[14] Amoruso, S., Bruzzese, R., Wang, X., Nedialkov, N. N. and Atanasov, P. A. "Femtosecond laser ablation of nickel in vacuum,” J. Phys. D: Appl. Phys., 40, 331-340 (2007).

[15] Jaleh, B., Torkamany, M. J., Golbedaghi, R., Noroozi, M., Habibi, S., Samavat, F., Jaberian Hamedan, V. and Albeheshti, L. "Preparation of Nickel Nanoparticles via Laser Ablation in Liquid and Simultaneously Spectroscopy," Adv. Mater. Res., 403, 4440-4444 (2012).

[16] Musaev, O. R., Yan, J., Dusevich, V., Wrobel, J. M. and Kruger, M. B. Ni "Nanoparticles Fabricated by Laser Ablation in Water," Appl. Phys. A, 116, 735-739 (2014).

[17] Bohren, C. F. and Huffmanm, D. R. [Absorption and Scattering of Light by Small Particles], Wiley, New York, 1998.

[18] Rakić, A. D., Djurišic, A. B., Elazar, J. M. and Majewski, M. L. "Optical Properties of Metallic Films for VerticalCavity Optoelectronic Devices,” Appl. Opt., 37, 5271-5283 (1998).

[19] Kreibig, U. and Vollmer, M. [Optical Properties of Metal Clusters], Springer, Berlin, 1995.

[20] Petrovykh, D. Y., Altmann, K. N., Hochst, H., Laubscher, M., Maat, S., Mankey, G. J. and Himpse, F. J. "Spindependent Band Structure, Fermi Surface, and Carrier Lifetime of Permalloy," Appl. Phys. Lett., 73, 3459-3461 (1998).

[21] Scaffardi, L. B. and Tocho, J. O. "Size dependence of refractive index of Gold Nanoparticles," Nanotechnology, $17,1309-1315$ (2006).

[22] Mendoza Herrera, L. J., Muñetón Arboleda, D., Schinca, D. C. and Scaffardi, L. B. "Determination of Plasma Frequency, Damping Constant, and Size Distribution from the Complex Dielectric Function of Noble Metal Nanoparticles,” J. Appl. Phys., 116, 233105-233108 (2014).

[23] Liu, B., Hu, Z., Che, Y., Chen, Y. and Pan, X. "Nanoparticle Generation in Ultrafast Pulsed Laser Ablation of Nickel," Appl. Phys. Lett., 90, 044103-3 (2007).

[24] Khan, S. Z., Yuan, Y., Abdolvand, A., Schmidt, M., Crouse, P., Li, L., Liu, Z., Sharp, M. and Watkins, K. G. "Generation and Characterization of NiO Nanoparticles by Continuous Wave Fiber Laser Ablation in Liquid," J. Nanopart. Res., 11, 1421-1427 (2009). 